\title{
Electron in a tangled chain: multifractality at the small-world critical point
}

\author{
J. Quintanilla \\ School of Physics $\&$ Astronomy, University of Birmingham, \\ Edgbaston, Birmingham, West Midlands, B15 2TT, U.K. \\ ISIS facility, CCLRC Rutherford Appleton Laboratory, \\ Chilton, Didcot, Oxfordshire, OX11 OQX, U.K. \\ V. L. Campo \\ Departamento de Física e Informática, Instituto de Física de São Carlos, \\ Universidade de São Paulo, Caixa Postal 369, 13560-970 São Carlos, São Paulo, Brazil. \\ Centro Internacional de Física da Matéria Condensada, \\ Universidade de Brasília, Caixa Postal 04513, 70910-970, Brasília, Brazil.
}

\begin{abstract}
We study a simple model of conducting polymers in which a single electron propagates through a randomly tangled chain. The model has the geometry of a small-world network, with a small density $p$ of crossings of the chain acting as shortcuts for the electron. We use numerical diagonalisation and simple analytical arguments to discuss the density of states, inverse participation ratios and wave functions. We suggest that there is a critical point at $p=0$ and demonstrate finite-size scaling of the energy and wave functions at the lower band edge. The wave functions are multifractal. The critical exponent of the correlation length is consistent with criticality due to the small-world effect, as distinct from the previously discussed, dimensionality-driven Anderson transition.
\end{abstract}

\section{Introduction}

The small-world effect occurs in complex networks [1] that resemble regular lattices with a small density $p$ of "shortcuts" per site [2]. The key observable is the shortest path length between two randomly chosen points, $l$. Defining a "thermodynamic limit" by

$$
L \rightarrow \infty, p=\text { constant },
$$

where $L$ is the number of sites, one finds that for $0<$ $p \ll 1$ the network has the local structure of a regular lattice but some global properties of a random graph [2]. In fact there is a critical point [3, 4] at $p=0$ It separates regular lattices $(p=0)$, for which the relative size of the shortest path length, $l / L$, tends to some finite value, from lattices with finite density of shortcuts $(p>0)$, for which $l / L \rightarrow 0$. This quantity shows finite-size scaling $[3,4,[5,6]$ : for

$$
L \gg 1, p \ll 1
$$

it depends only on the scaling variable $L / \xi$, where $\xi=$ $p^{-\nu}$ is a critical system size diverging algebraically at $p=0$. A simple renormalisation group argument yields the critical exponent $\nu[3]$.

On the other hand it has been accepted for some time that conducting polymers must be thought of as complex networks [7]. Models have been studied in which a onedimensional Anderson insulator is perturbed by adding some additional connectivity in the form of a given density of chain "crossings". It is well established that, when the density of crossings reaches a finite critical value, which depends on the amount of site-energy disorder, there is an Anderson metal-insulator transition [7, [8, 9].
This critical point results from the increase of the effective dimensionality of the network. It has also been demonstrated [10, 11] in a model having the geometry of the Watts-Strogatz (WS) small-world network [2].

Interestingly, even models of conducting polymers that lack site-energy disorder, so that the state of a single wire with no crossings is metallic, display non-trivial behaviour suggestive of proximity to a second-order phase transition [12, 13]. But there has been no detailed discussion of the nature of this critical point or its location on the phase diagram. Here we shall provide evidence that it occurs when the density of crossings reaches zero and that its origin is the small-world effect.

The rest of paper is organised as follows. First we introduce a simple model that generalises the "winding chain" of Ref. [12] and has the geometry of the NewmanWatts (NW) network [3], with the crossings acting as shortcuts. Then we present results of full-spectrum numerical diagonalisation that demonstrate the small-world effect: a qualitative change of the spectrum and wave functions on the addition of a small density of shortcuts. We then use Lanczos diagonalisation to study in detail, for much larger systems, the lower band edge. We find finite-size scaling consistent with the critical behaviour of the network described in [3, 4]. Simple analytical arguments are used to characterise the wave functions at the critical point. Our conclusions are then laid out.

\section{Model}

We consider a single particle moving in a tight-binding lattice with one orbital per site. The site energy is equal on all $L$ sites. Hopping can take place between near- 
est neighbours or between sites connected by one of $N$ shortcuts. For simplicity we assume the same value of the hopping integral, $t$. The Hamiltonian is

$$
\hat{H}=-t \sum_{j}|j\rangle\left\langle j+1\left|-t \sum_{s=1}^{N}\right| i_{s}\right\rangle\left\langle j_{s}\right|+\text { H.c. }
$$

where $j$ is a site label and $s$ denotes the shortcut connecting sites $i_{s}$ and $j_{s}$. We assume periodic boundary conditions: $|L+1\rangle=|1\rangle$.

Note that $t$ is the only energy scale in the Hamiltonian (3): the system's behaviour is thus completely specified by the way in which the $N$ shortcuts are chosen. One can imagine creating these shortcuts by winding an initially straight chain, embedded in a higher-dimensional space, in a random fashion. Sites that were initially far apart may come close so tunnelling of the electron between these sites becomes possible.

The model of conducting polymers in Ref. [12] is obtained by requiring that such winding is in the form of a series of consecutive, non-intersecting loops: $i_{1}<j_{1}<$ $i_{2}<j_{2}<\ldots<i_{N}<j_{N}$ (except for the boundary conditions, which do not affect our argument). Here we relax that restriction: our chain is "tangled" at random so that each of

$$
\mathcal{N} \equiv \frac{L(L-3)}{2}
$$

possible shortcuts (excluding only shortcuts joining a site to itself or to a nearest neighbour) is present with the same probability,

$$
\mathcal{P} \equiv \frac{2 p}{(L-3)}
$$

The resulting topology is an instance of the NW network with critical exponent $\nu=1$ [27].

A similar Hamiltonian, namely the adjacency matrix of the WS network, has been considered in [14, 15]. The authors of [15] solved the time-dependent Schrödinger equation, demonstrating the faster spread of initially localised wave functions on a small-world network compared to a regular lattice. In [14] the eigenvalues and eigenvectors were obtained, as a means of characterising the networks' geometry. Our argument is based on a similar calculation. Note, however, that our model has a distinct topology, compatible with the simple picture of a tangled chain-indeed the Hamiltonian of [14, 15] is not of the form (3). Moreover our specific aim is to identify critical behaviour near $p=0$ [28].

In our model the number of shortcuts, $N$, is a random variable following the binomial distribution (Poisson for $L \gg p)$. Evidently $\langle N\rangle=L p$, where $\langle\ldots\rangle$ denotes a configurational average.

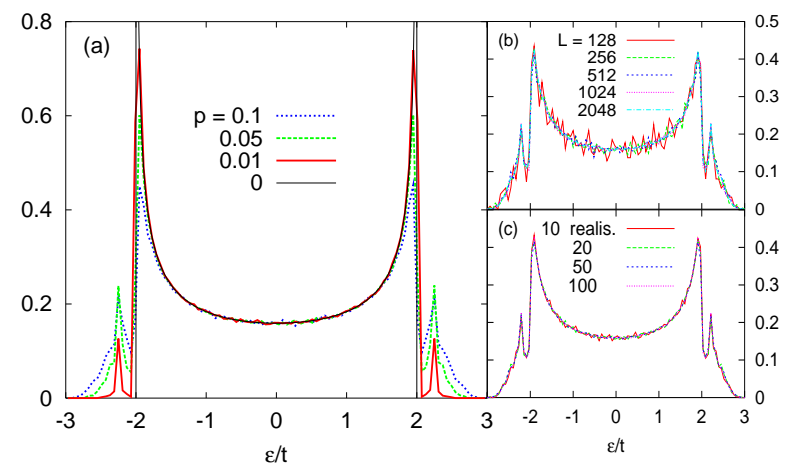

Figure 1: (Color online) Density of states (DOS), estimated from full-spectrum numerical diagonalisation for (a) $R=32$ realisations, $L=1024$ sites and different densities of shortcuts per site, $p$; (b) $R=16, p=0.1$ and different values of $L$; (c) different $R$ with $L=1024$ and $p=0.1$. All the plots have been obtained by drawing histograms using 100 uniformly spaced values of the energy from $\epsilon=-3 t$ to $\epsilon=+3 t$.

\section{Small-world effect}

For a finite size $L$ and a given set of shortcuts (a "realisation") it is straightforward to diagonalise the Hamiltonian (3) numerically [29]. One can then, by averaging over a finite number of realisations, $R$, obtain a coarsegrained estimate of the configurationally averaged density of states (DOS), $\rho(\epsilon) \equiv\left\langle\sum_{\nu} \delta\left(\epsilon-\epsilon_{\nu}\right)\right\rangle$, for a particular value of $p$. Here $\epsilon_{\nu}$ is the energy of the $\nu^{\text {th }}$ state.

The main panel of Fig. 1 shows the DOS, estimated in this way, for several small values of $p$. The curve for $p=0$, given by the well-known formula $\rho(\epsilon)=$ $\left(2 \pi \sqrt{1-\epsilon^{2} / 4 t^{2}}\right)^{-1}$, is also shown. Panels (b) and (c) illustrate the dependences of the numerical results on $L$ and on $R$. They suggest that the DOS is well defined in the thermodynamic limit (1), and that the plot on the main panel is representative of it, at this level of coarse-graining (higher values of $L$ and $R$ would be required to describe the finer detail). At $p=0$, the DOS has the usual square-root singularities at the band edges. For $p \gtrsim 0$, the singularities survive in the form of pronounced maxima in the DOS. This is similar to what was described for the model based on the WS network [14]. Also in common with that model, for $p \gtrsim 1$ (not shown), the spectral density resembles that obtained by numerical diagonalisation of the adjacency matrices of uncorrelated random graphs. However the salient features of Fig. 1 are two additional peaks outside the conduction band of the chain, located at $\epsilon= \pm \sqrt{5} t$. Although this region of the spectrum becomes less populated as $p \rightarrow 0$ the position of the peaks does not change and they are present for any non-zero value of $p$. They are a qualitative feature distinguishing the DOS for $p=0$ from that for $p \gtrsim 0$.

To explore further the nature of the states around these new peaks we can use numerical diagonalisation 


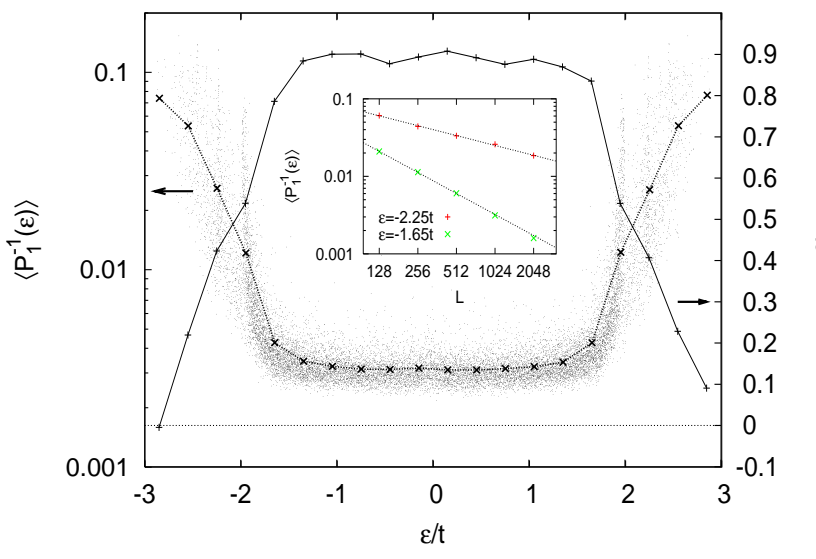

Figure 2: (Color online) Energy-dependence of the IPR (lefthand axis) and the exponent $\alpha_{1}$ characterising its power-law dependence on system size $L$ (right-hand axis). The data have been obtained by exact numerical diagonalisation for $R=16$ realisations with $p=0.1$ shortcuts/site. The IPR vs energy curve corresponds to $L=1024$ sites. It has been obtained by splitting the energy interval from $\epsilon=-3 t$ to $\epsilon=+3 t$ into 20 sub-intervals and averaging the IPR over each subinterval. The 16384 individual values of the IPR in our ensemble are also shown. Similar curves have been obtained for different values of $L$, and fits of the averaged IPR to a power-law in each energy interval have been used to determine $\alpha_{1}$. Two examples of such fits are shown in the inset.

to obtain the inverse participation ratio (IPR; $n=1$ ) and higher-order moments of the local density distribution $(n=2,3, \ldots)$. These are given by $P_{n}^{-1}(\epsilon) \equiv$ $\sum_{j \nu} \delta\left(\epsilon-\epsilon_{\nu}\right)\left|\psi_{\nu}(j)\right|^{2(n+1)}$ where $\psi_{\nu}(j)$ is the wave function of the $\nu^{\text {th }}$ state, evaluated on the $j^{\text {th }}$ site. For large enough values of $L$, we expect the configurationallyaveraged IPR to be given by a power law: $\left\langle P_{1}^{-1}(\epsilon)\right\rangle=$ $L^{-\alpha_{1}}$. The coefficient $\alpha_{1}$ can be used to distinguish extended states $\left(\alpha_{1}=1\right)$ from localised states $\left(\alpha_{1}=0\right)$ and states with fractal dimension $\left(0<\alpha_{1}<1\right)$ [16]. Fig. 2 shows our results. They suggest that, for $p=0.1$, all states are fractal, but the wave functions with $|\epsilon|>2 t$ are much closer to localisation than those with $|\epsilon|<2 t$, which are almost extended. Note the strong fluctuations of the IPR at particular energies.

The above results reflect that there are two quite different types of states. It is easy to show that the real eigenfunctions of (3) have the form

$$
\psi(x)=B_{l} e^{-i K\left(x-x_{l}\right)}+C_{l} e^{i K\left(x-x_{l}\right)}
$$

for $x_{l}<x<x_{l+1}$, where $x_{l}$ denotes the $l^{\text {th }}$ shortcut terminal (i.e. $x_{l}=i_{s}$ or $j_{s}$ for some $s$ ), ordered by ascending site index, and $x_{l+1}-x_{l}>2$. The parameter $K$ and the corresponding energy $\epsilon_{K}$ can only take one of the following forms: $K=-i \kappa \Rightarrow \epsilon_{K}=-2 t \cosh \kappa ; K=k \Rightarrow \epsilon_{K}=$ $-2 t \cos k ; K=\pi-i \kappa \Rightarrow \epsilon_{K}=2 t \cosh \kappa$. Thus in the space between shortcut terminals states with $|\epsilon|<2 t$ are plane waves, with wave number $k=\cos ^{-1}(-\epsilon / 2 t)$, while
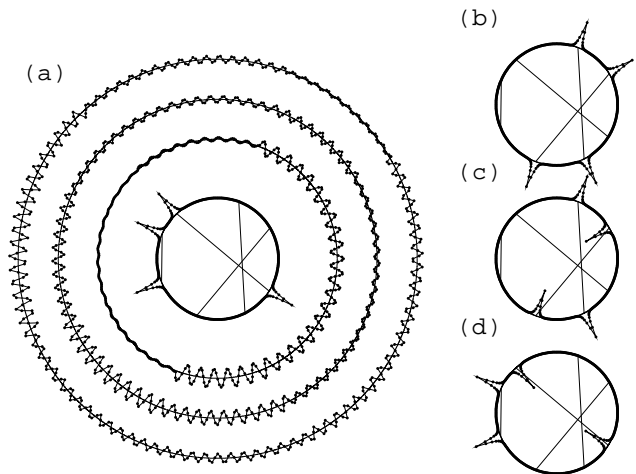

Figure 3: Numerically determined single-particle wave functions for a particular realisation of our tight-binding model, with $L=460$ sites. Each wave function has been represented on a circle, whose radius is its energy, measured from $-3 t$. The innermost wave function in (a) is the ground state. The three excited states with energy below $-2 t$ have been plotted separately for clarity: (b), (c) and (d), in order of increasing energy. The other wave functions in (a) have energies in $(-2 t, 0)$. Finally, the straight lines in the innermost circles indicate which sites are joined by "shortcuts".

those with $\pm \epsilon>2 t$ decrease or grow exponentially at the rate $\kappa=\cosh ^{-1}( \pm \epsilon / 2 t)$. A particular case is the ground state of a large chain with a large, single loop, which has energy $\epsilon=-\sqrt{5} t$ and is exponentially localised at the two shortcut terminals, with localisation length $\kappa^{-1} \sim 2$ sites [17]. The case with more than one shortcut terminal is more complex. A few wave functions for a particular realisation of our model, with $p \sim 0.01$ shortcuts per site, are shown in Fig. 3. States with $\epsilon<-2 t$ are exponentially localised on some of the shortcuts. Their energy is very close to $-\sqrt{5} t$, but the degeneracy is broken by localising on different sets of shortcuts and by forming bonding and antibonding combinations between them. This leads to the broadening of the peaks in Fig. 1. States with $-2 t<\epsilon<0$ resemble plane waves, except that their amplitude "jumps" at some of the shortcut terminals.

\section{Critical behaviour at the lower band edge}

The results presented above indicate a qualitative change of behaviour when $p$ becomes non-zero. In particular the gradual population of the energy peaks at $\epsilon= \pm \sqrt{5} t$ is suggestive of a critical point at $p=0$ in the thermodynamic limit (1). This further implies the universal dependence of all observables on $L / \xi$ in the finite-size scaling regime (2). The latter has been verified extensively for geometrical features of the NW and WS networks, such as the shortest path length and the clustering coefficient [3, 4, 5, , 6]. Here we are concerned with spectral properties and wave functions. To obtain data above and below the critical system size $\xi=p^{-1}$ we need to consider very large systems. In this case it is 


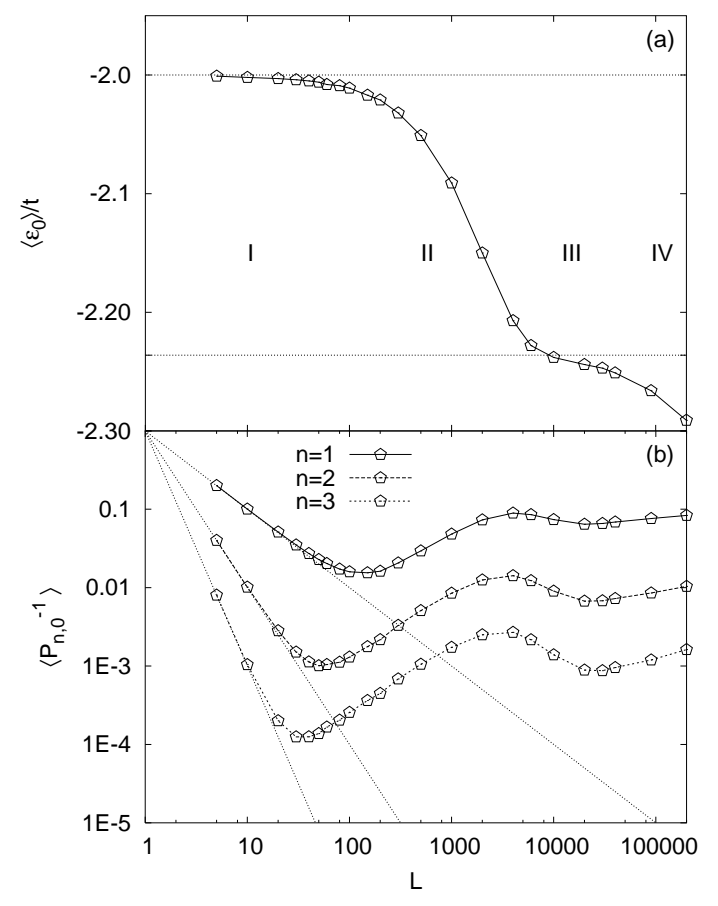

Figure 4: Dependence on the system size $L$ of (a) the energy and (b) the IPR $(n=1)$ and next two higher moments of the density distribution $(n=2,3)$ at the lower band edge obtained by Lanczos diagonalisation and averaged over 2000 random realisations. The density of shortcuts is $p=5 \times 10^{-4}$. The dotted lines indicate $\left\langle\epsilon_{0}\right\rangle=-2 t$ and $-\sqrt{5} t$ (a) and the analytical results for $p=0(\mathrm{~b})$.

best to use a sparse-matrix numerical algorithm [14]. We employ [18] which, for real symmetric matrices, is an implementation of the Lanczos method. For computational economy, given that we are now going to examine much larger system sizes, we concentrate on the single state at the lower band edge.

Fig. 4 shows, for fixed $p$, the dependence on $L$ of the configurational averages of the ground-state energy $\epsilon_{0}$ and of the moments of the density distribution for the ground state wave function, $P_{n, 0}^{-1} \equiv \sum_{j}\left|\psi_{0}(j)\right|^{2(n+1)}$. For finite conducting chains at fixed chemical potential, the average of $\epsilon_{0}$ gives a gross idea of how much the energy levels leak out, due to the presence of the shortcuts, below the bottom of the original conduction band. Our main interest in this quantity, however, is that it displays critical behaviour quantitatively consistent with the small-world effect (see below). There are four distinct regimes: for $L \ll \xi(\mathrm{I})$ most realisations do not contain any crossings, so $\left\langle\epsilon_{0}\right\rangle \approx-2 t$ and $\left\langle P_{n, 0}^{-1}\right\rangle=L^{-n}$. For $L \lesssim \xi$ (II) the system is in a crossover region in which $\left\langle\epsilon_{0}\right\rangle$ decreases and the $\left\langle P_{n, 0}^{-1}\right\rangle$ increase with system size. For $L \gtrsim \xi$ (III) the energy reaches a plateau and stays close to $\left\langle\epsilon_{0}\right\rangle \approx-\sqrt{5} t$. In this regime most realisations have one or more shortcuts, but their terminals are quite far apart. The ground-state wave functions look

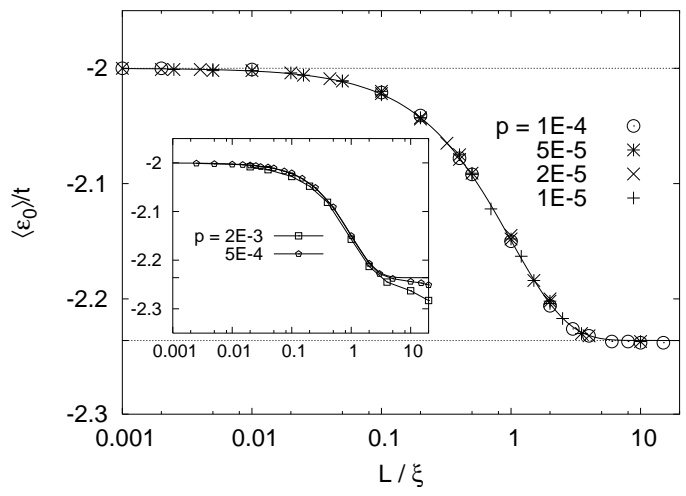

Figure 5: Finite-size scaling of the ground state energy, obtained as in Fig. 4 The main plot shows the collapse of the data for $p \leq 10^{-4}$ on the curve given by Eq. (8) for values of the scaling variable from $L / \xi=0.001$ to $L / \xi=20$. The two dotted lines indicate $\epsilon_{0}=-2 t$ and $\epsilon_{0}=-\sqrt{5} t$. The inset shows data for two higher values of $p$, for comparison.

like the one in Fig. 3 (a). Interestingly in this range of values of $L$ the $\left\langle P_{n, 0}^{-1}\right\rangle$ decrease again. This is because although locally the wave function is exponentially localised on shortcut terminals, globally the crossings on which it chooses to localise can be quite spread over the network, and such spread becomes greater as the network grows in size and more and more crossings become available. Finally for $L \gg \xi$ (IV) $\left\langle\epsilon_{0}\right\rangle$ decreases and the $\left\langle P_{n, 0}^{-1}\right\rangle$ turn to rise again. This indicates that in the thermodynamic limit (11) the lower band edge is at $-\infty$ and contains localised states, consistent with the Lifshitz tails suggested by Fig. 1 .

Figs. [5 and 6 show $\left\langle\epsilon_{0}\right\rangle$ and the $\left\langle P_{n, 0}^{-1}\right\rangle(n=1,2,3)$ for very low values of $p$, plotted as functions of the scaling variable $L / \xi=L p$. For $p \leq 10^{-4}$ crossings per site the data collapse to a single curve in the ranges of values of $L / \xi$ shown. The inset to Fig. 5 and Fig. 6 (b) show the same data for two higher values of $p$. Evidently the collapse is not so good. However there is a range of values of $L / \xi$ over which the data depend only on that variable which becomes wider as $p$ is lowered. Clearly this widening finite-size scaling regime corresponds to the regions II and III identified above.

The defining feature of the finite-size scaling regime is that, although the number of shortcuts on the network is arbitrary (indeed $\langle N\rangle=L / \xi$ ), all shortcut terminals are well separated. The probability of this is

$$
P_{\text {sep }} \approx \exp \left[-4 p(2 L p-1) \kappa^{-1}\right]
$$

(taking $N=p L$ and using that the relevant length scale is $4 \kappa^{-1} \ll L$; see the Appendix). Evidently in the thermodynamic limit (1) $P_{\text {sep }} \rightarrow 0$. On the other hand the finite-size scaling regime is reached when, for a given value of the scaling variable $L p$, the density of crossings $p$ is sufficiently low that $P_{\text {sep }} \approx 1$. In this case shortcuts are not close enough to lift the degeneracy of 

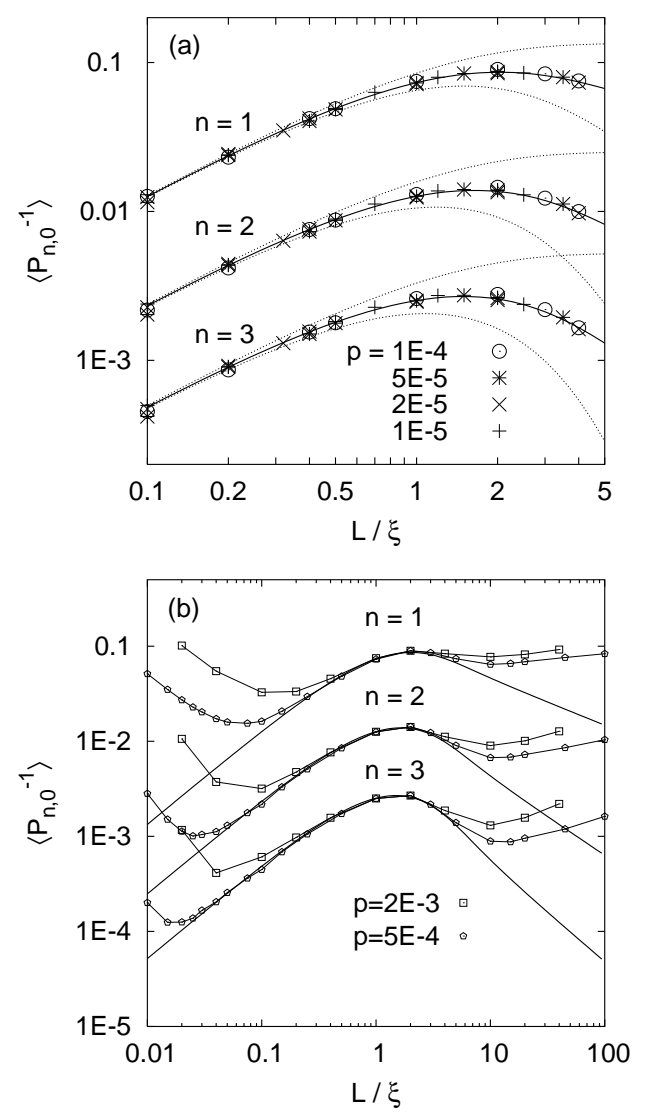

Figure 6: Finite-size scaling of the IPR $(n=1)$ and next two higher moments of the local density distribution $(n=2,3)$ at the lower band edge, obtained as in Fig. 4. (a) Shows the collapse of the data for $p \leq 10^{-4}$ on the curve given by Eq. (10) for values of the scaling variable from $L / \xi=0.1$ to $L / \xi=5$. The solid lines correspond to $\alpha=0.48,0.40$ and 0.33 and fit the $n=1,2,3$ data, respectively. The dotted lines correspond to $\alpha=0$ and $\alpha=1$. In (b) data for two higher values of $p$ over a wider range of values of $L / \xi$ is displayed for comparison.

the ground state and so the only possible energies are $\epsilon_{0}=-2 t$ (when there are no shortcuts) and $\epsilon_{0}=-\sqrt{5} t$ (when there are one or more). Since the probability of the former is $(1-\mathcal{P})^{\mathcal{N}} \approx e^{-\mathcal{P N}}$ (for $p \ll L$ ), with $\mathcal{N}$ and $\mathcal{P}$ defined in Eqs. (4) and (5) respectively, using $\mathcal{P N}=p L$ we obtain the following exact analytical expression for the finite-size scaling law giving the configurational average of the ground-state energy:

$$
\left\langle\epsilon_{0}\right\rangle=e^{-L / \xi}(-2 t)+\left(1-e^{-L / \xi}\right)(-\sqrt{5} t) .
$$

This is the curve plotted in Fig. [5alongside the numerical data. Let us now deploy a similar argument for the moments $\left\langle P_{n, 0}^{-1}\right\rangle$. Suppose that, for a given realisation, having $N$ shortcuts, the wave function were exponentially localised around a single site $x_{0}: \psi_{0}(x) \sim e^{-\kappa\left|x-x_{0}\right|}$. Then the moments at the lower band edge would be given by $P_{n, 0}^{-1}=\tanh ^{n+1} \kappa / \tanh \kappa(n+1)$. In actual fact the wave function is localised around $2 N^{\prime}$ sites, corresponding to the terminals of $N^{\prime} \leq N$ shortcuts. Since the sites $x_{t}$ of the terminals are all well separated we can derive (see the appendix)

$$
P_{n, 0}^{-1}=\frac{\tanh ^{n+1} \kappa}{\left[\tanh \kappa(n+1) 2^{n} N^{\prime n}\right]} .
$$

Let us assume that $N^{\prime}$ depends only on $N$ and postulate a power law: $N^{\prime}=N^{\alpha}$. Then using the Poisson distribution we obtain

$$
\left\langle P_{n, 0}^{-1}\right\rangle=\sum_{N=1}^{\infty} \frac{e^{-L / \xi}(L / \xi)^{N}}{N !} \frac{\tanh ^{n+1} \kappa}{\tanh \kappa(n+1)} \frac{1}{2^{n} N^{\alpha n}}
$$

which gives the finite-size scaling of the moments $\left\langle P_{n, 0}^{-1}\right\rangle$. It is important to note however that this expression, unlike (8), contains an adjustable parameter $\alpha$. Depending on its value it can describe very different behaviours. Indeed for $\alpha=1$ (i.e. requiring that the wave function is exponentially localised always on the same fraction of the shortcuts) we have

$$
\left\langle P_{1,0}^{-1}\right\rangle=\frac{\tanh ^{n+1} \kappa}{2 \tanh \kappa(n+1)}\{\operatorname{Ei}(L / \xi)-\ln (L / \xi)-\gamma\},
$$

where $\gamma$ is Euler's constant and $\operatorname{Ei}(x)$ is the exponential integral. For large $L / \xi$, we have $\left\langle P_{1,0}^{-1}\right\rangle \sim(L / \xi)^{-1}$ (more generally $\left.\left\langle P_{n, 0}^{-1}\right\rangle \sim(L / \xi)^{-n}\right)$ corresponding to a state which, although exponentially localised at the local level, is extended globally over the whole network. Conversly for $\alpha=0$ (i.e. the wave function is exponentially localised always on a single shortcut) we obtain $\left\langle P_{n, 0}^{-1}\right\rangle \approx$ constant for large $L / \xi$, i.e. the wave function is localised in the true sense of the word. The dashed lines in Fig. 6 (a) represent these two extreme cases. On the other hand the data are fitted quite accurately by using three different intermediate values of $\alpha$ for $n=1,2,3$, respectively. This is represented by the solid curves in Fig. 6. It suggests that the global structure of the wave function at the lower band edge is multifractal in the finite-size scaling regime.

\section{Conclusion}

In summary we have studied a simple model of conducting polymers in which chain crossings act as shortcuts for the electrons, so they move in a small-world topology. Our results are based on numerical diagonalisation (full spectrum for systems of size $L \sim 10^{3}$ and Lanczos for $L \sim 10^{5}$ ) and some simple analytical arguments. We have seen that a small density of shortcuts $p$ leads to qualitative, but continuous changes in the DOS and wave functions. The former take the form of new peaks appearing at well defined positions $\epsilon= \pm \sqrt{5} t$ outside the initial conduction band, $|\epsilon|<2 t$. At these peaks, 
the wave functions have a non-trivial structure. We have investigated in detail the lower band edge, confirming through finite-size scaling that there is a critical point at $p=0$. The critical exponent of the correlation length is consistent with criticality due to the small-world effect. We have derived analytical expressions for the scaling laws of the energy and the IPR and higher moments of the density distribution. The latter expressions contain an adjustable parameter $\alpha$ describing the global structure of the wave function, which we find to be multifractal.

We end by making two additional remarks. Firstly, the critical point described here is quite distinct from the dimensionality-driven Anderson transition considered before [7, 8, 9, 10, 11]. The latter occurs at a finite critical density of crossings $p_{c}(W)>0$, which depends on the amount of site-energy disorder $W$. Since real highly conducting polymers are intrinsically disordered [19] the small-world critical point at $p=0$ is not directly accessible to experiments. Nevertheless it may make a significant contribution to conductance fluctuations in the metallic state. Secondly, the multifractality of the wave functions suggests that the adjacency matrices of smallworld networks might be describable by the power law banded matrix ensemble [20] of random matrix theory. This intriguing possibility remains largely unexplored [30].

We thank P.R.A. Campos, K. Capelle, E.C. Carter, J.M.F. Gunn, C. Hooley, V.E. Kravtsov, I.V. Lerner, B.N. Narozhny, L.N. Oliveira, A.J. Schofield, R.A. Smith and I.V. Yurkevich for useful discussions. JQ thanks the Abdus Salam ICTP for hospitality during the preparation of part of this manuscript. We acknowledge financial support from CAPES (VLC), FAPESP and The Leverhulme Trust (JQ). In addition, JQ gratefully acknowledges an Atlas fellowship granted by CCLRC in association with St. Catherine's College in the Univeristy of Oxford.

\section{APPENDIX}

In this Appendix we provide some simple derivations omitted in the main text. Firstly, let us prove that the probability $P_{\text {sep }}$ of all the $2 N$ shortcut terminals being well separated from each other is given by Eq. (7). Each terminal is found at a random location along the chain. Let $x_{t}$, with $t=1,2, \ldots, 2 N$, be the site indices of those locations. As noted in the main text, the ground-state wave function decays exponentially as $e^{-\kappa\left|x-x_{t}\right|}$ in the space between terminals. After selecting the location of the first terminal, the probability for at least one of the $2 N-1$ remaining terminals to be within $2 \kappa^{-1}$ of $x_{1}$ (the factor of two is arbitrary and does not affect the argument) is $\left(4 \kappa^{-1} / L\right)(2 N-1)$. Thus the probability that the first terminal is separated form all the other terminals (in the sense that it is within two decay lengths of any one of them) is

$$
P_{1}=1-\frac{4 \kappa^{-1}}{L}(2 N-1) .
$$

Now consider the second terminal, located at $x_{2}$. The probability of the remaining $2 N-2$ terminals being well separated from this one, in the same sense as above, is $1-\left(4 \kappa^{-1} / L\right)(2 N-2)$. So the probability of both the first and second terminals being well separated from all the others is

$$
P_{2}=\left[1-\frac{4 \kappa^{-1}}{L}(2 N-1)\right]\left[1-\frac{4 \kappa^{-1}}{L}(2 N-2)\right] .
$$

At this point we can already see that the probability of all the terminals being well separated from each other is given by

$$
P_{\text {sep }}=P_{2 N-1}=\prod_{j=1}^{2 N-1}\left[1-\frac{4 \kappa^{-1}}{L}(2 N-j)\right]
$$

whence, taking logarithms,

$$
\log \left(P_{\mathrm{sep}}\right) \approx-\frac{4 \kappa^{-1}}{L} \sum_{j=1}^{2 N-1} 2 N-j=-\frac{4 \kappa^{-1} N}{L}(2 N-1) .
$$

Considering that the averaged number of shortcuts is $p L$, Eq.(7) follows.

Let us now prove Eq. (9) for the moments of the density distribution of the ground-state wave function. With $N$ well separated shortcuts, let us suppose the ground-state wave function has peaks at $2 N^{\prime}$ shortcut terminals $\left(N^{\prime} \leq\right.$ $N$ ), decaying exponentially from each maximum again as $e^{-\kappa\left|x-x_{t}\right|}$. The moment $P_{n, 0}^{-1}$ is given by

$$
\begin{aligned}
P_{n, 0}^{-1} & =\sum_{j=1}^{L}\left|\psi_{0}(j)\right|^{2 n+2} \\
& \approx|A|^{2 n+2} \sum_{t=1}^{2 N^{\prime}}\left[\sum_{j=-\infty}^{\infty}\left(e^{-\kappa|j|}\right)^{2 n+2}\right] \\
& =|A|^{2 n+2} \frac{2 N^{\prime}}{\tanh [(n+1) \kappa]},
\end{aligned}
$$

where $A$ is a normalization constant. The latter can be found from $P_{0,0}^{-1}=1$. It is

$$
A^{2}=\frac{\tanh (\kappa)}{2 N^{\prime}}
$$

which substituted in Eq. (16) yields Eq. (9).

[1] R. Albert and A.-L. Barabási, Rev. Mod. Phys. 74, 47 (2002). 
[2] D. J. Watts and S. H. Strogatz, Nature 393, 440 (1998).

[3] M. Newman and D. Watts, Phys. Lett. A 263, 341 (1999).

[4] M. Newman and D. Watts, Phys. Rev. E 60, 7332 (1999).

[5] M. Bathélémy and L. A. N. Amaral, Phys. Rev. Lett. 82, 3180 (1999), erratum: 82, 5180 (1999).

[6] A. Barrat and M. Weigt, Eur. Phys. J. B 13, 547 (2000).

[7] V. Prigodin and K. Efetov, Phys. Rev. Lett. 70, 2932 (1993).

[8] I. Zambetakiy, S. N. Evangelou, and E. N. Economou, J. Phys. : Condens. Matter 8, L605 (1996).

[9] N. Dupuis, Phys. Rev. B 56, 3086 (1997).

[10] C.-P. Zhu and S.-J. Xiong, Phys. Rev. B 62, 14780 (2000).

[11] C.-P. Zhu and S.-J. Xiong, Phys. Rev. B 63, 193405 (2001).

[12] S.-J. Xiong and S. Evangelou, Phys. Rev. B 52, R13079 (1995).

[13] S.-J. Xiong, Y. Chen, and S. N. Evangelou, Phys. Rev. Lett. 77, 4414 (1996).

[14] I. J. Farkas, I. Derenyi, A. L. Barabasi, and T. Vicsek, Phys. Rev. E 64, 026704 (2001).

[15] B. J. Kim, H. Hong, and M. Y. Choi, Phys. Rev. B 68, 014304 (2003).

[16] B. Kramer and A. MacKinnon, Rep. Prog. Phys. 56 (1993).

[17] Y. M. Abrukina and B. Oxsengendler, 60, 270 (1994).

[18] R. Lehoucq, D. Sorensen, and C. Yang, ARPACK library.
http://www.caam.rice.edu/software/ARPACK

[19] A. J. Heeger, S. Kivelson, J. R. Schrieffer, and W. P. Su, Rev. Mod. Phys. 60, 781 (1988).

[20] V. E. Kravtsov and K. A. Muttalib, Phys. Rev. Lett. 79, 1913 (1997).

[21] B. Luque and O. Miramontes, cond-mat/0211383.

[22] R. Metzler et al., Phys. Rev. Lett. 88, 188101 (2002).

[23] X. R. Bao, H. J. Lee, and S. R. Quake, Phys. Rev. Lett. 91, 265506 (2003).

[24] R. Monasson, Eur. Phys. J. B 12, 555 (1999).

[25] B. J. Kim, H. Hong, and M. Y. Choi, J. Phys. A: Math. Gen. 36, 6329 (2003).

[26] J. W. Eaton et al., Octave package. http://www.octave.org

[27] One can envisage more realistic generalisations of the model in [12] that take into account correlations between the positions of different crossings [21, 22] or their unquenched nature [23]. Our choice of model is dictated by simplicity and by analogy with the Anderson model (which features quenched, uncorrelated site-energy disorder).

[28] A related class of models, describing vibrations of tangled chains, results when the Laplace operator is defined on the NW [24] or WS networks [25].

[29] We used the diagonalisation function of [26].

[30] We acknowledge V. E. Kravtsov and B. N. Narozhny for this suggestion. 